\title{
From screening to confinement in a Higgs-like model
}

\author{
Patricio Gaete ${ }^{1}$ \\ Departamento de Física, Universidad Técnica Federico Santa María, Valparaíso, \\ Chile \\ Euro Spallucci ${ }^{2}$ \\ Dipartimento di Fisica Teorica, Università di Trieste and INFN, Sezione di \\ Trieste, Italy
}

\begin{abstract}
We investigate a recently proposed Higgs-like model (arXiv:0811.4423 [hep-th]), in the framework of a gauge-invariant but path-dependent variables formalism. We compute the static potential between test charges in a condensate of scalars and fermions.

In the case of charged massive scalar we recover the screening potential. On the other hand, in the Higgs case, with a "tachyonic" mass term and a quartic potential in the Lagrangian, unexpected features are found. It is observed that the interaction energy is the sum of an effective-Yukawa and a linear potential, leading to the confinement of static charges.
\end{abstract}

\section{Introduction}

It is a well known fact that, despite an intense effort for more than three decades, a full understanding of the QCD vacuum structure and color confinement mechanism are still lacking. String theory $A d S / C F T$ duality [1] is the most promising technical framework to face non-perturbative $Q C D$ effects. Despite an intensive activity in this advanced research sector, a final word is still to come. In the meanwhile, phenomenological models still offer a proper platform for understanding different features of the physics of confinement. In

1 e-mail address: patricio.gaete@usm.cl

2 e-mail address: spallucci@ts.infn.it 
this context it may be useful to recall that the phenomenon of condensation has provided valuable insight and theoretical guidance into this problem. For instance, in the illustrative picture of dual superconductivity [2,3]4, where it is conjectured that the QCD vacuum behaves as a dual-type II superconductor. More explicitly, because of the condensation of magnetic monopoles, the chromoelectric field acting between $q \bar{q}$ pair is squeezed into strings, and the nonvanishing string tension represents the proportionality constant in the linear potential. In passing let us also mention a theory of antisymmetric tensor fields that results from the condensation of topological defects $[5,6,7,8,9]$ as a consequence of the Julia-Toulouse mechanism, which also leads to confinement of static charges [10].

Condensation of charged scalars and its physical consequences such as Friedel oscillations and the strong screening of an electric charge have created a lot of interest in recent times [11 12,13]. Much of this work has been motivated by the possibility of describing condensed helium- 4 nuclei in an electron background in white dwarf cores. In particular, a Lorentz-violating Higgs-like effective Lagrangian has been considered. The crucial ingredient of this development is to introduce a nonzero vacuum expectation value for the fermion field which permits to realize the condensation of the helium-4. As a result, it was shown a strong screening between probe charges placed in the condensate. It should be noted that the condensate characterizes the new vacuum of the theory with striking consequences over the different phases of the pure gauge sector of the proposed model. Given the relevance of these studies, it is of interest to enrich our understanding of the physical consequences presented by condensates. Thus, our purpose here is to further explore the impact of condensates on physical observables. Of special interest will be to provide an additional independent demonstration on the screening in the condensate, based in a Hamiltonian framework.

As we mentioned above, our concern in this work is to provide a different calculation of the interaction energy between pointlike sources in the framework of the recently proposed Lorentz-violating Higgs-like effective model [14,15]. To this end we will use the gauge-invariant but path-dependent variables formalism [16], which is a physically-based alternative to the Wilson loop approach. When we compute in this way the static potential for the model described in [14], in the case of a "right-sign" mass term $m_{H}^{2} \phi^{*} \phi$, we obtain an effective Yukawa potential, which in the $m_{\gamma} \ll M$ approximation is identical to the one encountered in [14]. On the other hand, in the case of a "wrong-sign" mass term $-m_{H}^{2} \phi^{*} \phi$, the result of this calculation is new and rather unexpected. We show that the interaction energy is the sum of an effective -Yukawa and a linear potential, leading to the confinement of static charges. It is interesting to note that the above static profile is analogous to that encountered in both Abelian and non-Abelian models. Remarkable examples are: theory of antisymmetric tensor fields that results from the condensation of topological 
defects as a consequence of the Julia-Toulouse mechanism [10]; gauge theory with a pseudoscalar coupling, in the case that there is a constant magnetic strength expectation value [17]; gauge theory which includes the mixing between the familiar photon $U(1)_{Q E D}$ and a second massive gauge field living in the so-called hidden-sector $U(1)_{h}$ [18. On the non-Abelian side, we find the case of gluodynamics in curved space-time [19], and of non-Abelian gauge theory with a mixture of pseudoscalar and scalar couplings, in the case where a constant chromoelectric, or chromomagnetic, strength expectation value is present [20]. These connections thus provide us with a new kind of "duality" among diverse models and allow us to exploit this equivalence in concrete calculations, as we are going to illustrate.

\section{Scalar condensation}

We turn now to the problem of obtaining the interaction energy between static point-like sources for a Lorentz-violating Higgs-like effective model. To do this, we shall compute the expectation value of the energy operator $H$ in the physical state $|\Phi\rangle$ describing the sources, which we will denote by $\langle H\rangle_{\Phi}$. However, before going to the derivation of the interaction potential, we will describe the salient features of this model in the familiar language of standard quantum field theory. For this purpose we restrict our attention to scalar electrodynamics in the presence of a fermion background density:

$$
\mathcal{L}=-\frac{1}{4} F_{\mu \nu}^{2}+\left|D_{\mu} \phi\right|^{2}-m_{H}^{2} \phi^{*} \phi+\bar{\psi}\left(i \gamma^{\mu} D_{\mu}-M_{f}\right) \psi
$$

$\phi$ is a charged, massive, scalar field; $A_{\mu}$ is a $U(1)$ gauge potential and $\psi$ is an "heavy" fermion. The covariant derivative is defined as usual

$$
D_{\mu} \equiv \partial_{\mu}-i e A_{\mu}
$$

Let us remark that $m_{H}^{2}>0$ is a "right sign" mass term and we have not included any self-interaction for the scalar field. Indeed, the condensation mechanism we are going to consider is quite different from spontaneous symmetry breaking and relies on the presence of a non-trivial fermion density in the ground state. In order to implement this setting we suppose fermions to be so heavy that they cannot be excited in the low energy regime we are considering. Thus, the Dirac kinetic term can be neglected and the whole fermion sector of the model reduces to a constant background density $J^{0}$ coupled to $A_{\mu}$

$$
\bar{\psi} \gamma^{\mu} \psi \longrightarrow \delta_{0}^{\mu} J^{0}
$$


In such a case,

$$
\mathcal{L} \rightarrow-\frac{1}{4} F_{\mu \nu}^{2}+\left|D_{\mu} \phi\right|^{2}-m_{H}^{2} \phi^{*} \phi+e J^{0} \delta_{0}^{\mu} A_{\mu}
$$

In Eq.(4) $J^{\mu}$ acts as an "external source" for the time component of the gauge potential. From this point of view, the fermionic condensate breaks Lorentz invariance by giving a constant vacuum expectation value to $A_{0}$ as we are going to show in a while.

The field equations obtained by varying (44) with respect to $A_{\mu}$ and $\phi^{*}$ read

$$
\begin{aligned}
& \partial_{\mu} F^{\mu \nu}+2 e^{2} A^{\nu}|\phi|^{2}=e J_{s}^{\nu}+e J^{0} \delta_{0}^{\nu} \\
& \left(D_{\mu} D^{\mu}+m_{H}^{2}\right) \phi=0
\end{aligned}
$$

where $J_{s}^{\nu} \equiv i \phi^{*} \partial^{\nu} \phi-i \phi \partial^{\nu} \phi^{*}$.

The presence of a homogeneous source $J^{0}$ allows the existence of constant classical solutions of the form

$$
\begin{aligned}
& \phi=\phi^{*}=\text { const. } \equiv \phi_{0} \neq 0, \\
& A_{\mu} \equiv \frac{\mu_{s}}{e} \delta_{\mu}^{0} .
\end{aligned}
$$

The two integration constants $\phi_{0}, \mu_{s}$ are determined by inserting (7), (8) in (5), (6) :

$$
\begin{aligned}
& 2 e^{2} A^{\nu} \phi_{0}^{2}=e J^{0} \delta_{0}^{\nu}, \\
& \left(e^{2} A^{2}-m_{H}^{2}\right) \phi_{0}=0 .
\end{aligned}
$$

Eq.(15) and (8) determine $\mu_{s}$

$$
A^{2}=\frac{m_{H}^{2}}{e^{2}}=\frac{\mu_{s}^{2}}{e^{2}} \longrightarrow \mu_{s}=m_{H}
$$

Eq.([6) and (77) fix $\phi_{0}$

$$
A_{\mu}=\frac{J^{0}}{2 e \phi_{0}^{2}} \delta_{\mu 0}=\frac{m_{H}}{e} \delta_{\mu 0} \longrightarrow \phi_{0}=\sqrt{\frac{J^{0}}{2 m_{H}}}
$$

In summary, the ground state of the system is described by the classical solution: 


$$
\begin{aligned}
& \bar{\psi}_{0} \gamma^{\mu} \psi_{0}=\delta_{0}^{\mu} J^{0}, \\
& \phi_{0}=\sqrt{\frac{J^{0}}{2 m_{H}}}, \\
& A_{(0) \mu}=\frac{m_{H}}{e} \delta_{\mu}^{0} .
\end{aligned}
$$

While fermions are "frozen" into the ground state, both $\phi$ and $A_{\mu}$ are subject to quantum fluctuations. More exactly, the modulus of $\phi$ oscillates and its phase has been gauged to zero. By splitting fields into a background value and a dynamical part:

$$
\begin{aligned}
& \phi=\phi^{*}=\phi_{0}+\frac{1}{\sqrt{2}} \eta(x), \\
& A_{\mu}=\frac{m_{H}}{e} \delta_{\mu}^{0}+b_{\mu}(x),
\end{aligned}
$$

we expand the Lagrangian up to quadratic terms in the fluctuations

$$
\mathcal{L}^{(2)}=-\frac{1}{4} f_{\mu \nu}^{2}+\frac{1}{2}\left(\partial_{\mu} \eta\right)^{2}+\frac{1}{2} m_{\gamma}^{2} b_{\mu}^{2}+2 m_{H} m_{\gamma} b_{0} \eta .
$$

Here $f_{\mu \nu} \equiv \partial_{\mu} b_{\nu}-\partial_{\nu} b_{\mu}$ and $m_{\gamma}^{2} \equiv 2 e^{2} \phi_{0}^{2}$.

It is important to remark that the scalar fluctuation $\eta$ is massless due to a cancelation between the original mass term $m_{H}^{2}$ and a quadratic term from the gauge covariant kinetic term. In the case of the Higgs field the same two terms sum up leading to massive fluctuations and a different static potential. We are going to discuss this case in the last part of the paper.

Next, integrating out the $\eta$ field induces an effective theory for the $b_{\mu}$ field, that is,

$$
\mathcal{L}=-\frac{1}{4} f_{\mu \nu}^{2}+\frac{1}{2} m_{\gamma}^{2} b_{\mu}^{2}+2 m_{H}^{2} m_{\gamma}^{2} b_{0} \frac{1}{\Delta} b_{0},
$$

which is a Maxwell-Proca-like theory with a manifestly Lorentz violating term. It can be worth to remark that the mixing of such a term with the Lorentz invariant ones is the key element to reproduce the correct screening potential. In order to restore the gauge invariance in (19), we shall use the Hamiltonian formalism for constrained systems because it leads directly to a physical Hamiltonian, as we are going to illustrate in the next section.

\section{Interaction energy}

As already mentioned, our immediate objective is to calculate the interaction energy between external probe sources for the model under consideration. To 
do this, our first undertaking is to restore the gauge invariance in (19). For this we shall use the Hamiltonian formalism for constrained systems along the lines of Ref. [21]. To achieve this end we first note that the Lagrangian (19) may be rewritten as

$$
\mathcal{L}=-\frac{1}{4} f_{\mu \nu}^{2}+\frac{1}{2} b_{\mu} m^{2} b^{\mu}-\frac{1}{2} b_{i} \frac{\left(2 m_{H} m_{\gamma}\right)^{2}}{\Delta} b^{i}
$$

where $m^{2} \equiv m_{\gamma}^{2}\left(1+\frac{4 m_{H}^{2}}{\Delta}\right)$. The canonically conjugate are $\Pi^{0}=0$ and $\Pi^{i}=$ $-f^{0 i}$. This leads us to the canonical Hamiltonian,

$H=\int d^{3} x\left\{-b_{0}\left(\partial_{i} \Pi^{i}+\frac{m^{2}}{2} b^{0}\right)-\frac{1}{2} \Pi_{i} \Pi^{i}+\frac{1}{4} f_{i j} f^{i j}-\frac{1}{2} b_{i}\left(m^{2}-\frac{\left(2 m_{H} m_{\gamma}\right)}{\Delta}\right) b^{i}\right\}$,

Requiring the primary constraint $\Pi^{0}=0$ to be preserved in time yields the following secondary constraint

$$
\Gamma(x) \equiv \partial_{i} \Pi^{i}+m^{2} b^{0}=0 .
$$

The above result reveals the second class nature of the constraints, as expected for a theory with an explicit mass term which breaks the gauge invariance. Next, as was explained in Ref. [21], we enlarge the original phase space by introducing a canonical pair of fields $\theta$ and $\Pi_{\theta}$. Accordingly, a new set of constraints can be defined in this extended space:

$$
\Lambda_{1} \equiv \Pi_{0}+m^{2} \theta
$$

and

$$
\Lambda_{2} \equiv \Gamma+\Pi_{\theta} .
$$

It is simple to see that the new constraints are first class and in this way restore the gauge symmetry of the theory under consideration. Therefore the new effective Lagrangian, after integrating out the $\theta$ field, reads

$$
\mathcal{L}=-\frac{1}{4} f_{\mu \nu}\left[1+\frac{m_{\gamma}^{2}}{\Delta}\left(1+\frac{4 m_{H}^{2}}{\Delta}\right)\right] f^{\mu \nu} .
$$

We observe that to get this new theory we have ignored the last term in (20) because it add nothing to the static potential calculation, as we will show it below. In other words, this new effective theory provide us with a suitable starting point to study the interaction energy.

Having characterized the theory under study, we can now compute the interaction energy. To obtain the corresponding Hamiltonian, we must carry out the quantization of this theory. The Hamiltonian analysis starts with the computation of the canonical momenta $\Pi^{\mu}=-\left[1+\frac{m_{\gamma}^{2}}{\Delta}\left(1+\frac{4 m_{H}^{2}}{\Delta}\right)\right] f^{0 \mu}$, which pro- 
duces the usual primary constraint $\Pi^{0}=0$ and $\Pi^{i}=-\left[1+\frac{m_{\gamma}^{2}}{\Delta}\left(1+\frac{4 m_{H}^{2}}{\Delta}\right)\right] f^{0 i}$. The canonical Hamiltonian is then

$$
H_{C}=\int d^{3} x\left\{-b_{0} \partial_{i} \Pi^{i}-\frac{1}{2} \Pi_{i}\left[1+\frac{m_{\gamma}^{2}}{\Delta}\left(1+\frac{4 m_{H}^{2}}{\Delta}\right)\right]^{-1} \Pi^{i}+\frac{1}{4} f_{i j} f^{i j}\right\} .
$$

Time conservation of the primary constraint yields the usual Gauss constraint $\Gamma_{1}(x) \equiv \partial_{i} \Pi^{i}=0$. Note that the time stability of this constraint does not induce further constraints. Consequently, the extended Hamiltonian that generates translations in time then reads $H=H_{C}+\int d^{3} x\left(c_{0}(x) \Pi_{0}(x)+c_{1}(x) \Gamma_{1}(x)\right)$. Here $c_{0}(x)$ and $c_{1}(x)$ are arbitrary Lagrange multipliers. It should be noted that $\dot{b}_{0}(x)=\left[b_{0}(x), H\right]=c_{0}(x)$, which is an arbitrary function. Since $\Pi^{0}=0$ always, neither $b^{0}$ nor $\Pi^{0}$ are of interest in describing the system and may be discarded from the theory. Thus the Hamiltonian is now given as

$$
H=\int d^{3} x\left\{c(x) \partial_{i} \Pi^{i}-\frac{1}{2} \Pi_{i}\left[1+\frac{m_{\gamma}^{2}}{\Delta}\left(1+\frac{4 m_{H}^{2}}{\Delta}\right)\right]^{-1} \Pi^{i}+\frac{1}{4} f_{i j} f^{i j}\right\},
$$

where $c(x)=c_{1}(x)-b_{0}(x)$.

In order to break the gauge freedom of the theory, it is necessary to impose one gauge constraint such that the full set of constraints become second class. A particularly convenient choice is found to be

$$
\Gamma_{2}(x) \equiv \int_{C_{\xi x}} d z^{\nu} b_{\nu}(z) \equiv \int_{0}^{1} d \lambda x^{i} b_{i}(\lambda x)=0
$$

where $\lambda(0 \leq \lambda \leq 1)$ is the parameter describing the spacelike straight path $x^{i}=\xi^{i}+\lambda(x-\xi)^{i}$, and $\xi$ is a fixed point (reference point). There is no essential loss of generality if we restrict our considerations to $\xi^{i}=0$. The choice (28) leads to the Poincaré gauge [16]. As a consequence, we can now write down the only nonvanishing Dirac bracket for the canonical variables

$$
\left\{b_{i}(x), \Pi^{j}(y)\right\}^{*}=\delta_{i}^{j} \delta^{(3)}(x-y)-\partial_{i}^{x} \int_{0}^{1} d \lambda x^{j} \delta^{(3)}(\lambda x-y) .
$$

We have finally assembled the tools to determine the interaction energy for the model under consideration. As mentioned before, in order to accomplish this purpose we will calculate the expectation value of the energy operator $H$ in the physical state $|\Phi\rangle$. Now we recall that the physical state $|\Phi\rangle$ can be written as

$$
|\Phi\rangle \equiv\left|\bar{\Psi}(\mathbf{y}) \Psi\left(\mathbf{y}^{\prime}\right)\right\rangle=\bar{\psi}(\mathbf{y}) \exp \left(i q \int_{\mathbf{y}^{\prime}}^{\mathbf{y}} d z^{i} b_{i}(z)\right) \psi\left(\mathbf{y}^{\prime}\right)|0\rangle,
$$


where the line integral is along a spacelike path on a fixed time slice, and $|0\rangle$ is the physical vacuum state.

Now, using the previous Hamiltonian structure, and since the fermions are taken to be infinitely massive (static) we can substitute $\Delta$ by $-\nabla^{2}$ in Eq. (27). In such a case $\langle H\rangle_{\Phi}$ reduces to

$$
\langle H\rangle_{\Phi}=\langle H\rangle_{0}+\langle H\rangle_{\Phi}^{(1)}
$$

where $\langle H\rangle_{0}=\langle 0|H| 0\rangle$, and the $\langle H\rangle_{\Phi}^{(1)}$ term is given by

$$
\langle H\rangle_{\Phi}^{(1)}=\left\langle\Phi\left|\int d^{3} x\left\{-\frac{1}{2} \Pi_{i}\left[1-\frac{m_{\gamma}^{2}}{\nabla^{2}}\left(1-\frac{4 m_{H}^{2}}{\nabla^{2}}\right)\right]^{-1} \Pi^{i}\right\}\right| \Phi\right\rangle .
$$

The reason why we eliminated from the effective Lagrangian the term involving $b_{0}$ now becomes clear, that is, the commutator for the fields $b_{\mu}$ is zero. Next, it should be noted that expression (31) may be conveniently rewritten as

$$
\langle H\rangle_{\Phi}^{(1)}=-\frac{1}{2} \frac{4 M^{4}}{\left(M_{2}^{2}-M_{1}^{2}\right)} \int d^{3} x\left\langle\Phi\left|\Pi_{i}\left\{\alpha \frac{\nabla^{2}}{\left(\nabla^{2}-M_{1}^{2}\right)}-\beta \frac{\nabla^{2}}{\left(\nabla^{2}-M_{2}^{2}\right)}\right\} \Pi^{i}\right| \Phi\right\rangle,
$$

with $\alpha=\frac{1}{\left(M_{1}^{2}-m_{\gamma}^{2}\right)}$ and $\beta=\frac{1}{\left(M_{2}^{2}-m_{\gamma}^{2}\right)}$. While $M_{1}^{2}=\frac{1}{2}\left(m_{\gamma}^{2}+\sqrt{m_{\gamma}^{4}-16 M^{4}}\right)$, $M_{2}^{2}=\frac{1}{2}\left(m_{\gamma}^{2}-\sqrt{m_{\gamma}^{4}-16 M^{4}}\right)$ and $M \equiv \sqrt{m_{\gamma} m_{H}}$.

¿From our above Hamiltonian analysis we observe that $\langle H\rangle_{\Phi}^{(1)}$ takes the form

$$
\langle H\rangle_{\Phi}^{(1)}=\langle H\rangle_{\Phi}^{(1 a)}+\langle H\rangle_{\Phi}^{(1 b)}
$$

where the $\langle H\rangle_{\Phi}^{(1 a)},\langle H\rangle_{\Phi}^{(1 b)}$ terms are given by

$$
\begin{aligned}
\langle H\rangle_{\Phi}^{(1 a)} & =-\frac{\alpha}{2} \frac{4 M^{4}}{\left(M_{2}^{2}-M_{1}^{2}\right)} \int d^{3} x \int_{\mathbf{y}}^{\mathbf{y}^{\prime}} d z_{i}^{\prime} \delta^{(3)}\left(\mathbf{x}-\mathbf{z}^{\prime}\right)\left(1-\frac{M_{1}^{2}}{\nabla^{2}}\right)_{x}^{-1} \times \\
& \times \int_{\mathbf{y}}^{\mathbf{y}^{\prime}} d z^{i} \delta^{(3)}(\mathbf{x}-\mathbf{z}),
\end{aligned}
$$

and

$$
\begin{aligned}
\langle H\rangle_{\Phi}^{(1 b)} & =\frac{\beta}{2} \frac{4 M^{4}}{\left(M_{2}^{2}-M_{1}^{2}\right)} \int d^{3} x \int_{\mathbf{y}}^{\mathbf{y}^{\prime}} d z_{i}^{\prime} \delta^{(3)}\left(\mathbf{x}-\mathbf{z}^{\prime}\right)\left(1-\frac{M_{2}^{2}}{\nabla^{2}}\right)_{x}^{-1} \times \\
& \times \int_{\mathbf{y}}^{\mathbf{y}^{\prime}} d z^{i} \delta^{(3)}(\mathbf{x}-\mathbf{z}) .
\end{aligned}
$$


One immediately sees that these expressions are analogous to that encountered in previous works [10,17, 18, 19,20]. In view of this situation, we find that the potential for two opposite charges located at $\mathbf{y}^{\prime}$ and $\mathbf{y}$ becomes

$$
V=-\frac{q^{2}}{4 \pi} \frac{4 M^{4}}{\left(M_{2}^{2}-M_{1}^{2}\right)}\left[\frac{1}{\left(M_{1}^{2}-m_{\gamma}^{2}\right)} \frac{e^{-M_{1}\left|\mathbf{y}-\mathbf{y}^{\prime}\right|}}{\left|\mathbf{y}-\mathbf{y}^{\prime}\right|}-\frac{1}{\left(M_{2}^{2}-m_{\gamma}^{2}\right)} \frac{e^{-M_{2}\left|\mathbf{y}-\mathbf{y}^{\prime}\right|}}{\left|\mathbf{y}-\mathbf{y}^{\prime}\right|}\right]
$$

¿From this expression we see that, in the limit $m_{\gamma} \ll M$, the potential reduces to

$$
V=-\frac{q^{2}}{4 \pi} \frac{e^{-M\left|\mathbf{y}-\mathbf{y}^{\prime}\right|}}{\left|\mathbf{y}-\mathbf{y}^{\prime}\right|} \cos \left(M\left|\mathbf{y}-\mathbf{y}^{\prime}\right|\right)
$$

Expression (38) is identical to the one encountered in [14], which has been computed using the propagator of the theory. Thus one is led to the conclusion that the contributions of the gauge field propagator are properly captured in the gauge invariant formalism.

\section{Higgs confining phase}

In this final part of the paper we consider a charged scalar field with a "wrongsign" mass term and a quartic self-interaction potential. This is the simplest model where the Higgs mechanism can occur. The new effect we are going to study is the interplay between the Higgs vacuum and the fermion condensate,

$$
\mathcal{L}=-\frac{1}{4} F_{\mu \nu}^{2}+\left|D_{\mu} \phi\right|^{2}+m_{H}^{2} \phi^{*} \phi-\frac{\lambda}{6}\left(\phi^{*} \phi\right)^{2}+e J^{0} \delta_{0}^{\mu} A_{\mu}
$$

Notice that the self-interaction coupling constant is assumed to be positive, i.e. $\lambda>0$, in order to have a potential energy bounded from below. By setting

$$
\phi \equiv \frac{\sigma}{\sqrt{2}} e^{i \alpha}
$$

and choosing the unitary gauge $\alpha=0$, we get

$$
\mathcal{L}=-\frac{1}{4} F_{\mu \nu}^{2}+\frac{e^{2} A^{2} \sigma^{2}}{2}+\frac{1}{2}\left(\partial_{\mu} \sigma\right)^{2}+\frac{m_{H}^{2}}{2} \sigma^{2}-\frac{\lambda}{24} \sigma^{4}+e J^{0} \delta_{0}^{\mu} A_{\mu}+e J_{s}^{\nu} A_{\nu}
$$

The field equation obtained by varying (39) with respect to $A_{\mu}$ is the same as before while the scalar field equation acquires a new term 


$$
\begin{aligned}
& \partial_{\mu} F^{\mu \nu}+e^{2} A^{\nu} \sigma^{2}=e J_{s}^{\nu}+e J^{0} \delta_{0}^{\nu}, \\
& \left(\Delta+e^{2} A^{2}+m_{H}^{2}-\frac{\lambda}{6} \sigma^{2}\right) \sigma=0 .
\end{aligned}
$$

We look for a homogeneous classical solution, as we did in the previous case

$$
\begin{aligned}
& \sigma=\text { const. } \equiv \phi_{0} \neq 0, \\
& A_{\mu} \equiv \frac{\mu_{s}}{e} \delta_{\mu}^{0} .
\end{aligned}
$$

The two integration constants $\phi_{0}, \mu_{s}$ are determined by inserting (44), (45) in (42), (43):

$$
\begin{aligned}
& e^{2} A^{\nu} \phi_{0}^{2}=e J^{0} \delta_{0}^{\nu} \\
& \left(e^{2} A^{2}+m_{H}^{2}-\frac{\lambda}{6} \phi_{0}^{2}\right) \phi_{0}=0
\end{aligned}
$$

Eq.(46) and (45) determine $\mu_{s}$

$$
\mu_{s}^{3}+m_{H}^{2} \mu_{s}-\frac{\lambda}{6} J^{0}=0
$$

Eq.(44) and (47) fix $\phi_{0}$

$$
\phi_{0}^{2}=\frac{J^{0}}{\mu_{s}}
$$

To simplify calculation we consider the case $\mu_{s}<<m_{H}$. In this case we obtain

$$
\begin{aligned}
\mu_{s} & \approx \frac{\lambda J^{0}}{6 m_{H}^{2}}, \\
\phi_{0}^{2} & \approx \frac{6 m_{H}^{2}}{\lambda} .
\end{aligned}
$$

In summary, the ground state of the system is described by the classical solution: 


$$
\begin{aligned}
& \bar{\psi}_{0} \gamma^{\mu} \psi_{0}=\delta_{0}^{\mu} J^{0}, \\
& \phi_{0}^{2}=\frac{J^{0}}{\mu_{s}} \approx \frac{6 m_{H}^{2}}{\lambda}, \\
& A_{\mu}=\frac{\mu_{s}}{e} \delta_{\mu}^{0} \approx \frac{\lambda J^{0}}{6 e m_{H}^{2}} \delta_{\mu}^{0} .
\end{aligned}
$$

We write the fields as a Higgs vacuum expectation value and a quantum fluctuation:

$$
\begin{aligned}
& \sigma=\phi_{0}+\eta(x)=\sqrt{\frac{6 m_{H}^{2}}{\lambda}}+\eta(x), \\
& A_{\mu}=\frac{\mu_{s}}{e} \delta_{\mu}^{0}+b_{\mu}(x)=\frac{\lambda J^{0}}{6 e m_{H}^{2}} \delta_{\mu}^{0}+b_{\mu}(x) .
\end{aligned}
$$

Next, we expand the Lagrangian up to quadratic terms in the fluctuations around the Higgs vacuum

$$
\mathcal{L}^{(2)}=-\frac{1}{4} f_{\mu \nu}^{2}+\frac{1}{2} m_{\gamma}^{2} b_{\mu}^{2}+2 e \phi_{0} \mu_{s} b_{0} \eta+\frac{1}{2}\left(\partial_{\mu} \eta\right)^{2}-m_{H}^{2} \eta^{2}
$$

where $m_{\gamma}^{2} \equiv e^{2} \phi_{0}^{2} \approx 6 e^{2} m_{H}^{2} / \lambda$.

As we anticipated in a previous section the scalar fluctuation is now massive and integrating out the $\eta$ field induces a new effective theory for the $b_{\mu}$ field:

$$
\mathcal{L}=-\frac{1}{4} f_{\mu \nu}^{2}+\frac{1}{2} m_{\gamma}^{2} b_{\mu}^{2}+\frac{1}{2} b_{0} \frac{4 e^{2} \mu_{s}^{2} \phi_{0}^{2}}{\left(\Delta+2 m_{H}^{2}\right)} b_{0} .
$$

In the same way as was done in the previous case, one finds

$$
\begin{aligned}
\langle H\rangle_{\Phi} & =-\frac{\alpha}{2} \int d^{3} x\left\langle\Phi\left|\Pi_{i}\left\{\frac{1}{M_{1}^{2}} \frac{\nabla^{2}}{\left(\nabla^{2}-M_{2}^{2}\right)}-\frac{1}{M_{2}^{2}} \frac{\nabla^{2}}{\left(\nabla^{2}-M_{1}^{2}\right)}\right\} \Pi^{i}\right| \Phi\right\rangle \\
& +\frac{\beta}{2} \int d^{3} x\left\langle\Phi\left|\Pi_{i}\left\{\frac{1}{M_{1}^{2}} \frac{1}{\left(\nabla^{2}-M_{2}^{2}\right)}-\frac{1}{M_{2}^{2}} \frac{1}{\left(\nabla^{2}-M_{1}^{2}\right)}\right\} \Pi^{i}\right| \Phi\right\rangle,
\end{aligned}
$$

where $\alpha \equiv \frac{6 M^{4}}{\left(M_{2}^{2}-M_{1}^{2}\right)}, \beta \equiv \frac{\left(2 m_{H}^{2}\right) 6 M^{4}}{\left(M_{2}^{2}-M_{1}^{2}\right)}$.

While $M_{1}^{2}=\frac{1}{2}\left[\left(m_{\gamma}^{2}+2 m_{H}^{2}\right)+\sqrt{\left(m_{\gamma}^{2}+2 m_{H}^{2}\right)^{2}-24 M^{4}}\right]$,

$M_{2}^{2}=\frac{1}{2}\left[\left(m_{\gamma}^{2}+2 m_{H}^{2}\right)-\sqrt{\left(m_{\gamma}^{2}+2 m_{H}^{2}\right)^{2}-24 M^{4}}\right]$ and $M=\sqrt{m_{\gamma} m_{H}}$. 
Once again, following our earlier procedure [10]17,18,19,20], we see that the potential for two opposite charges located at $\mathbf{y}^{\prime}$ and $\mathbf{y}$ takes the form

$$
\begin{aligned}
V & =-\frac{q^{2}}{4 \pi} \alpha\left\{\frac{1}{M_{1}^{2}} \frac{e^{-M_{2}\left|\mathbf{y}-\mathbf{y}^{\prime}\right|}}{\left|\mathbf{y}-\mathbf{y}^{\prime}\right|}-\frac{1}{M_{2}^{2}} \frac{e^{-M_{1}\left|\mathbf{y}-\mathbf{y}^{\prime}\right|}}{\left|\mathbf{y}-\mathbf{y}^{\prime}\right|}\right\} \\
& +\frac{q^{2}}{8 \pi} \beta\left\{\frac{1}{M_{1}^{2}} \ln \left(1+\frac{\Lambda^{2}}{M_{2}^{2}}\right)-\frac{1}{M_{2}^{2}} \ln \left(1+\frac{\Lambda^{2}}{M_{1}^{2}}\right)\right\}\left|\mathbf{y}-\mathbf{y}^{\prime}\right|
\end{aligned}
$$

where $\Lambda$ is a short-distance cutoff. Here, in contrast to the previous case, unexpected features are found. Interestingly, it is observed that the $+m_{H}^{2} \phi^{*} \phi$ term induces a Yukawa piece plus a linear confining piece. Before going ahead, we would like to remark that the cutoff $\Lambda$ is a physical cutoff, so no ultraviolet divergence arises in (60). To understand why $\Lambda$ is a physical cutoff, we observe that the first expression inside the second term on the right hand side of Eq.(60) describes a flux tube, which is characterized by having a tension independent of its length. In fact, the electric field due to a charge leading to this flux is a constant electric field $\left(E_{0}\right)$. In other words, the energy stored in the flux tube is proportional to its length. This implies that $\Lambda^{2}=M_{2}^{2}\left(e^{\frac{8 \pi M_{1}^{2}}{q^{2} \beta}\left|E_{0}\right|}-1\right)$, which is finite.

\section{$5 \quad$ Final remarks}

In summary, we have considered the recently proposed Higgs-like model [14], which describes a condensed of charged scalars in a neutralizing background of fermions, from a somewhat different perspective. First, we have studied the phenomenon of charged scalars in the familiar language of standard quantum field theory. The internal consistency of this development was illustrated. Second, a Hamiltonian analysis of the effective theory was done in order to restore the gauge invariance. Third, based in the gauge-invariant but path-dependent variables formalism, we have examined the confinement versus screening issue for this new theory. When we compute in this way the static potential in the case of a "right-sign" mass term $m_{H}^{2} \phi^{*} \phi$, we obtain an effective - Yukawa potential, which in the $m_{\gamma} \ll M$ approximation is identical to the one encountered in [14]. On the other hand, in the case of a "wrong-sign" mass term $-m_{H}^{2} \phi^{*} \phi$, the result of this calculation is new and rather unexpected. We have showed that the interaction energy is the sum of an effective -Yukawa and a linear potential, leading to the confinement of static charges. As expressed in the Introduction, similar forms of interaction potentials have been reported before in different contexts. In this way a correspondence was established among diverse effective models. The extension of these results to diverse dimensions 
or to supersymmetric models would be welcome.

Acknowledgments

One of us (PG) wants to thank the Physics Department of the Università di Trieste for hospitality and INFN for support. This work was supported in part by Fondecyt (Chile) grant 1080260 (PG).

\section{References}

[1] J. M. Maldacena, "Lectures on AdS/CFT" Prepared for Theoretical Advanced Study Institute in Elementary Particle Physics (TASI 2002): Particle Physics and Cosmology: The Quest for Physics Beyond the Standard Model(s), Boulder, Colorado, 2-28 Jun 2002.

[2] Y. Nambu, Phys. Rev. D 10, 4262 (1974).

[3] S. Mandelstam, Phys. Rept. 23307 (1976).

[4] G. 't Hooft, "Gauge Theory For Strong Interactions " Lectures given at Int. School of Subnuclear Physics, 'Ettore Majorana', Erice, Sicily, Jul 11-31, 1975. Published in Erice Subnucl.Phys.1975:0261.

[5] K. Seo and A. Sugamoto, Phys. Rev. D 24, 1630 (1981).

[6] P. Mansfield, Nucl. Phys. B 267, 575 (1986).

[7] E. Guendelman and D. A. Owen, Phys. Lett. B 235, 313 (1990).

[8] F. Quevedo and C. A. Trugenberger, Int. J. Mod. Phys. A 12, 1227 (1997).

[9] S. Ansoldi, A. Aurilia, L. Marinatto and E. Spallucci, Prog. Theor. Phys. 103, 1021 (2000).

[10] P. Gaete and C. Wotzasek, Phys. Lett. B 601, 108 (2004).

[11] G. Gabadadze and R. A. Rosen, Phys. Lett. B 658, 266 (2008).

[12] G. Gabadadze and R. A. Rosen, Phys. Lett. B 666, 277 (2008).

[13] G. Gabadadze and R. A. Rosen, JCAP 0810, 030 (2008).

[14] G. Gabadadze and R. A. Rosen, "Effective Lagrangian and Quantum Screening in Charged Condensate," arXiv:0811.4423 [hep-th].

[15] A. D. Dolgov, A. Lepidi and G. Piccinelli, "Electrodynamics at non-zero temperature, chemical potential, and Bose condensate," arXiv:0811.4406 [hep-th].

[16] P. Gaete and I. Schmidt, Phys. Rev. D 61, 125002 (2000). 
[17] P. Gaete and E. I. Guendelman, Mod. Phys. Lett. A 20, 319 (2005).

[18] P. Gaete and I. Schmidt, "Confinement effects from massive photons" arXiv:0807.0816 [hep-th].

[19] P. Gaete and E. Spallucci, Phys. Rev. D 77, 027702 (2008).

[20] P. Gaete and E. Spallucci, J. Phys. A 41, 185401 (2008).

[21] P. Gaete, E. Guendelman and E. Spallucci, Phys. Lett. B644, 379 (2007). 